\title{
EMBEDDINGS OF DIFFERENTIAL OPERATOR RINGS AND GOLDIE DIMENSION
}

\author{
DECLAN QUINN
}

(Communicated by Donald Passman)

\begin{abstract}
The differential operator ring $S=R[x ; \delta]$ can be embedded in $A_{1}(R)$, the first Weyl algebra over $R$, where $R$ is a Q-algebra and $\delta$ is a locally nilpotent derivation on $R$. Furthermore $A_{1}(R)$ is again a differential operator ring over the image of $S$. We consider similar embeddings of the smash product $R \# U(L)$, where $L$ is a finite dimensional Lie algebra and $U(L)$ is its universal enveloping algebra. We show that the Weyl algebra over $R$ has the same Goldie dimension as $R$ itself and use this to prove that $R$ and $R[x ; \delta]$ have the same Goldie dimension where $R$ is again a $\mathbf{Q}$-algebra and $\delta$ is locally nilpotent.
\end{abstract}

Introduction. Let $\delta$ be a derivation on the ring $R$. The corresponding differential operator ring $S=R[x ; \delta]$ is an associative ring formed by taking polynomials in $x$ over $R$ under the usual addition and with multiplication subject to the rule $x r-r x=\delta(r)$ for all $r \in R$. In Theorem 2 we show that if $R$ is a $\mathbf{Q}$-algebra and $\delta$ is locally nilpotent then $S=R[x ; \delta]$ can be embedding in

$$
A_{1}(R)=\frac{R\langle X, Y\rangle}{\langle X Y-Y X-1\rangle}=R[Y][X ; d / d Y]
$$

the first Weyl algebra over $R$. This result is well known. For example it follows from an old result of Nouaze and Gabriel [4, Théorème 1.2] which gives conditions for $S=R[x ; \delta]$ itself to be isomorphic to a Weyl algebra. An explicit embedding, similar to that used here, has been found by T. Masuda [3, Lemma 3.4].

This embedding is then used to show that if $L$ is a finite dimensional nilpotent Lie algebra over a field $k$, of characteristic zero, which acts on $R$ as locally nilpotent derivations, then the smash product $R \# U(L)$ embeds in $A_{n}(R)$, where $U(L)$ is the universal enveloping algebra of $L$ and $n=\operatorname{dim}_{k} L$. The existence of such an embedding follows from [1, Corollary 4.4]. We also consider finite dimensional solvable Lie algebras acting as derivations and give a similar embedding with $A_{n}(R)$ replaced by a certain extension ring, $\bar{A}_{n}(R)$.

It was shown by $\mathrm{R}$. Shock [5] that $R$ and $R[x]$ have the same right Goldie dimension. Here we show that $\operatorname{dim} R=\operatorname{dim} A_{1}(R)$ and we use this to show that $\operatorname{dim} R[x ; \delta]=\operatorname{dim} R$, where $R$ is a $\mathbf{Q}$-algebra and $\delta$ is locally nilpotent.

Differential operator rings. Let $\delta$ be a locally nilpotent derivation on the Q-algebra $R$ and let $S=R[x ; \delta]$ be the corresponding differential operator ring. Define $\eta: R \rightarrow R[Y] \subseteq A_{1}(R)$ by $\eta(r)=\tilde{r}=\sum_{i}\left(\delta^{i}(r) / i !\right) Y^{i}$. Since $\delta$ is locally nilpotent, this is a finite sum. Let $\tilde{R}=\eta(R)$.

Received by the editors July 29, 1985 and, in revised form August 20, 1986.

1980 Mathematics Subject Classification (1985 Revision). Primary 16A03.

(C) 1988 American Mathematical Society $0002-9939 / 88 \$ 1.00+\$ .25$ per page 
LEMMA 1. $\eta: R \rightarrow R[Y] \subseteq A_{1}(R)$ is a ring embedding with $\eta(1)=1$. Furthermore $Y$ is transcendental over $\tilde{R}$ and $\tilde{R}[Y]=R[Y]$.

PROOF. If $r, s \in R$, then, $\eta(r+s)=\eta(r)+\eta(s)$ since $\delta^{n}(r+s)=\delta^{n}(r)+\delta^{n}(s)$ for all $n$. The coefficient of $Y^{k}$ in $\eta(r) \eta(s)$ is $\sum_{i=0}^{k} \delta^{i}(r) \delta^{k-i}(S) / i !(k-i) !=\delta^{k}(r s) / k !$, by Leibniz's rule. Thus $\eta(r) \eta(s)=\eta(r s)$. Note that the constant term of $\tilde{r}$ is $r$ so that $\eta$ is injective and, since $\delta(1)=0, \eta(1)=1$.

Now suppose $\tilde{r}_{0}+\tilde{r}_{1} Y+\cdots+\tilde{r}_{t} Y^{t}=0$ and consider this as a polynomial in $Y$ with coefficients from $R$. The constant term is $r_{0}$, so that $r_{0}=0$. If we know that $r_{0}, \ldots, r_{i-1}$ are all zero, then the coefficient of $Y^{i}$ is $r_{i}$, giving that $r_{i}$ is zero also. Thus $r_{0}=\cdots=r_{t}=0$, so that $Y$ is transcendental over $\tilde{R}$. Clearly, $\tilde{R}[Y] \subseteq R[Y]$. Conversely, $Y \in \tilde{R}[Y]$ and, if $r \in R$, then $r=\tilde{r}-\widetilde{\delta(r)} Y+\cdots+\left((-1)^{n} / n !\right) \delta^{n}(r) Y^{n}$, where $\delta^{n+1}(r)=0$, and hence $r \in \tilde{R}[Y]$. Thus $R[Y] \subseteq \tilde{R}[Y]$ so that equality holds.

If $A$ is any associative ring and $\alpha \in A$, then $\operatorname{ad}_{\alpha}(\beta)=[\alpha, \beta]=\alpha \beta-\beta \alpha$ for all $\beta \in A$. Note that if $r \in R$, we have $\tilde{r}, X \in A_{1}(R)$ and

$$
\operatorname{ad}_{X}(\tilde{r})=[X, \tilde{r}]=\sum_{i}\left(\delta^{i}(r) / i !\right)\left[X, Y^{i}\right]=\sum_{i}\left(\delta^{i}(r) / i !\right) i Y^{i-1}=\widetilde{\delta(r)} .
$$

We can now prove

THEOREM 2. Let $\delta$ be a locally nilpotent derivation on the $\mathbf{Q}$-algebra $R$. Then $S=R[x ; \delta]$ is isomorphic to $\tilde{R}[X]$ via the map taking $r$ to $\tilde{r}$ and $x$ to $X$. Furthermore $Y \in A_{1}(R)$ is transcendental over $\tilde{R}[X], \tilde{R}[X]$ is invariant under $\operatorname{ad}_{Y}$, and $A_{1}(R)=\tilde{R}[X][Y]$ is a differential operator ring over $\tilde{R}[X]$ with the locally nilpotent derivation $\operatorname{ad}_{Y}=-d / d X$.

ProOF. Since $\tilde{R} \subset R[Y], X$ is transcendental over $\tilde{R}$ and, from above, $[X, \tilde{r}]=$ $\widetilde{\delta(r)}$. Thus $\tilde{R}[X]$ is isomorphic to $R[x ; \delta]$.

Now suppose $\sum_{i=0}^{k} f_{i} Y^{i}=0$ where $f_{i} \in \tilde{R}[X]$ and $f_{j} \neq 0$ for some $j$. Let $l=\max _{i} X-\operatorname{deg} f_{i}$ and assume $l$ is minimal over all such equations. Applying $\operatorname{ad}_{Y}$ we have that $0=\left[Y, \sum_{i=0}^{k} f_{i} Y^{i}\right]=-\sum_{i=0}^{k}\left(\partial f_{i} / \partial X\right) Y^{i}$. By the minimality of $l, \partial f_{i} / \partial X=0$ for each $i$, so that $f_{i} \in \tilde{R}$. But $Y$ is transcendental over $\tilde{R}$ by Lemma 1 and hence $Y$ is also transcendental over $\tilde{R}[X]$.

Finally, since $\operatorname{ad}_{Y}=-d / d X$, it is clear that $\operatorname{ad}_{Y}$ is a locally nilpotent derivation when restricted to $\tilde{R}[X]$. Also $R[Y] \subseteq \tilde{R}[X][Y]$ and $X \in \tilde{R}[X] \subseteq \tilde{R}[X][Y]$. Thus $\tilde{R}[X][Y]=A_{1}(R)$.

Now let $\bar{A}_{1}(R)=R[[Y]][X ; d / d Y]$, where $R[[Y]]$ is the ring of formal power series in $Y$ over $R$ and $d / d Y$ denotes formal differentiation of power series with respect to $Y$. Note that $A_{1}(R)=R[Y][X ; d / d Y] \subseteq \bar{A}_{1}(R)$.

If we drop the assumption that $\delta$ is locally nilpotent, $S=R[x ; \delta]$ may still be embedded in $\bar{A}_{1}(R)$ when $R$ is a $\mathbf{Q}$-algebra. Here we let $\eta: R \rightarrow R[[Y]]$ be given by $\eta(r)=\tilde{r}=\sum_{i}\left(\delta^{i}(r) / i !\right) Y^{i}$ where this sum is now allowed to be infinite. As before, $\eta$ is a ring monomorphism with $\eta\left(1_{R}\right)=1_{R}$.

It makes sense to take power series in $Y$ with coefficients from $\tilde{R} \subseteq R[[Y]]$, since in the sum $\sum_{i=0}^{\infty} \tilde{r}_{i} Y^{i}$, the coefficient of each power of $Y$ receives only finitely many contributions. We write the set of these elements $\tilde{R}[[Y]]$. 
LEMMA 3. In $\tilde{R}[[Y]], \sum_{i} \tilde{r}_{i} Y^{i}=\sum_{i} \tilde{s}_{i} Y^{i}$ if and only if $r_{i}=s_{i}$ for each $i$. Furthermore $\tilde{R}[[Y]]=R[[Y]]$.

PROOF. Suppose $\sum_{i} \tilde{r}_{i} Y^{i}=\sum_{i} \tilde{s}_{i} Y^{i}$ and consider both sides as power series in $Y$ with coefficients from $R$. Comparing constant terms gives that $r_{0}=s_{0}$. If we have verified that $r_{i}=s_{i}$ for $i=0,1, \ldots, n-1$, then considering the coefficients of $Y^{n}$ gives that $r_{n}=s_{n}$. Thus by induction, $r_{i}=s_{i}$ for all $i$.

It is clear that $\tilde{R}[[Y]] \subseteq R[[Y]]$ and since the constant term of $\tilde{r}$ is $r$, it is easy to see that any element of $R[[Y]]$ can be inductively constructed as a power series in $Y$.over $\tilde{R}$, so that the reverse inclusion holds.

The following result is the analogue of Theorem 2 for $\bar{A}_{1}(R)$.

THEOREM 4. Let $\delta$ be a derivation on the $\mathbf{Q}$-algebra $R$. Then $S=R[x ; \delta]$ is isomorphic to $\tilde{R}[X] \subseteq \bar{A}_{1}(R)$ via the map taking $r$ to $\tilde{r}$ and $x$ to $X$. Furthermore $\tilde{R}[[Y]][X]=R[[Y]][X]$.

PROOF. The isomorphism $R[x ; \delta] \simeq \tilde{R}[X]$ is proved as before and since $\tilde{R}[[Y]]=$ $R[[Y]]$, it follows that $\tilde{R}[[Y]][X]=R[[Y]][X]$.

We remark that $R[[Y]][X] \neq R[X][[Y]]$ since the elements of $R[[Y]][X]$ have finite $X$-degree. It can be shown that $R[[Y]][X] \subseteq R[X][[Y]]$ and consists of the elements of $R[X][[Y]]$ with finite $X$-degree.

If $R$ is a $Q$-algebra, it is known that the ideals of $A_{1}(R)$ are generated by their intersection with $R$ [2, Satz 4.10]. Thus the map taking $I \triangleleft R$ to $A_{1}(I) \triangleleft A_{1}(R)$, is a bijection between the ideals of $R$ and those of $A_{1}(R)$. If $R$ is right Noetherian a similar result holds for $\bar{A}_{1}(R)$. First we require a lemma.

LEMMA 5. Let $R$ be a Q-algebra and let $I \subseteq R[[Y]]$ be a finitely generated right ideal of $R[[Y]]$. Then $I=(I \cap R) R[[Y]]$ if and only if $I$ is closed under $d / d Y$.

ProOF. If $I=(I \cap R) R[[Y]]$, it is clear that $I$ is closed under $d / d Y$.

Now suppose $I$ is closed under $d / d Y$ and let $K_{t}=\left\{a_{t} \mid \sum_{i} a_{i} Y^{i} \in I\right\}$. Since $I$ is closed under multiplication by $Y, K_{i} \subseteq K_{i+1}$. Also $I$ is closed under $d / d Y$ and $R$ is a Q-algebra, so that $K_{i+1} \subseteq K_{i}$. Thus $K_{t}=K_{0}$ for all $t$ and $K_{0}$ is a right ideal of $R$. It follows that $I \subseteq K_{0}[[Y]]$.

Let $\phi_{1}, \ldots, \phi_{n}$ generate $I$ as a right ideal of $R[[Y]]$. Note that the constant terms of $\phi_{1}, \ldots, \phi_{n}$ must generate $K_{0}$ as a right ideal of $R$. A simple inductive argument now shows that $\phi_{1}, \ldots, \phi_{n}$ generate $K_{0}[[Y]]$ as a right ideal of $R[[Y]]$. Thus $I=K_{0}[[Y]]$ and since we have shown that $K_{0}$ is finitely generated as a right ideal of $R$,

$$
I=K_{0}[[Y]]=K_{0} R[[Y]]=(I \cap R) R[[Y]] .
$$

We now give the ideal correspondence between $R$ and $\bar{A}_{1}(R)$ when $R$ is Noetherian. If $I$ is an ideal of $R$, let $\bar{A}_{1}(I)$ be the ideal of $\bar{A}_{1}(R)$ consisting of the elements of $\bar{A}_{1}(R)$ whose coefficients lie in $I$. If $T$ is any $\operatorname{ring}$, let $I(T)$ denote the set of two-sided ideals of $T$.

LEMMA 6. Let $R$ be a right Noetherian Q-algebra. Then $\phi: I(R) \rightarrow I\left(\bar{A}_{1}(R)\right)$, where $\phi(I)=\bar{A}_{1}(I)$, is a bijection. This map preserves sums, products, and intersections. Also primes are sent to primes and primitives to primitives.

ProOF. Suppose $J \triangleleft \bar{A}_{1}(R)$. We need to show $J=\bar{A}_{1}(I)$ for some $I \triangleleft R$. Let $\alpha=\sum_{i=0}^{n} f_{i} X^{i} \in J$, where $f_{i} \in R[[Y]]$. Note that $\operatorname{ad}_{Y} \alpha=-\sum_{i=1}^{n} f_{i} i X^{i-1} \in J$, 
and so, by induction on the $X$-degree of $\alpha$, we can conclude that $f_{i} \in J$ for each $i>0$, and then, that $f_{0} \in J$. Thus $J=K[X]$, where $K=J \cap R[[Y]] . K$ is finitely generated as a right ideal of $R[[Y]]$ since $R$, and hence $R[[Y]]$, is right Noetherian. Also $K$ is invariant under $\operatorname{ad}_{X}=d / d Y$ so that by Lemma $5, K=I[[Y]]$ where $I=K \cap R$. Thus $J=K[X]=\bar{A}_{1}(I)$.

If $I, J \triangleleft R$, it is clear that $I J=\phi(I) \phi(J) \cap R$. Thus $\phi(I) \phi(J)=\bar{A}_{1}(I J)=\phi(I J)$. Similarly $\phi(I+J)=\phi(I)+\phi(J)$ and $\phi(I \cap J)=\phi(I) \cap \phi(J)$. Now since $\phi$ preserves products and inclusions, it is clear that $\phi$ sends primes to primes. To show that primitives go to primitive, let $P \triangleleft R$ be the annihilator of the simple right $R$-module $M$ and let $M[[Y]]=M \otimes_{R} R[[Y]] . R[[Y]]$ is a right $R[[Y]]$-module and this action can be extended to $\bar{A}_{1}(R)$ by letting $f \cdot X=-d f / d Y$ for each $f \in R[[Y]]$. It is easily checked that $R[[Y]]$ is now an $\left(R-\bar{A}_{1}(R)\right)$-bimodule so that $M[[Y]]=M \otimes_{R} R[[Y]]$ becomes a right $\bar{A}_{1}(R)$-module. Fix $m$ to be a nonzero element of $M$. Since $M$ is simple any element of $M[[Y]]$ can be written in the form $m \otimes f$ for some $f \in R[[Y]]$. Let $V$ be a nonzero $R[[Y]]$-submodule of $M[[Y]]$ and take $I$ to be the right ideal of $R[[Y]]$ given by $I=\{f \in R[[Y]] \mid m \otimes f \in V\} . \quad R[[Y]]$ is right Noetherian and $V$ is an $\bar{A}_{1}(R)$-submodule of $M[[Y]]$, so that $I$ is a finitely generated right ideal of $R[[Y]]$. Thus, by Lemma $5, I$ is generated as a right ideal of $R[[Y]]$ by its intersection with $R$. Let $J=I \cap R$. Then $V$ is generated as an $\bar{A}_{1}(R)$-module by $\{m \otimes j \mid j \in J\}=\{m j \otimes 1 \mid j \in J\}=M \otimes 1$, since $M$ is a simple $R$-module. Now $V=M[[Y]]$ so that $M[[Y]]$ is a simple $\bar{A}_{1}(R)$-module. Since the annihilator of $M[[Y]]$ is an ideal of $\bar{A}_{1}(R)$, it is of the form $\bar{A}_{1}(I)$ for some $I \triangleleft R$. Note that the right annihilator of $M=M \otimes 1 \subset M[[Y]]$ in $R$ is $P$, so that $I \subset P$. Conversely, since $P$ is finitely generated as a right ideal of $R$, it is clear that $P$ annihilates $M[[Y]]$ and then, that $\bar{A}_{1}(P)$ is the annihilator of the simple module $M[[Y]]$.

The following example, due to Passman, shows that Lemma 5 fails if $I$ is not assumed to be finitely generated. This also shows that we cannot drop the Noetherian hypothesis in Lemma 6.

EXAMPLE 7. Let $k$ be a field of characteristic zero and let

$$
R=k+t k[x] \subseteq k\left[x, t \mid t^{2}=0\right]
$$

Also let $I$ be the ideal of $R[[Y]]$, invariant under $d / d Y$, generated by $t e^{x Y}$. Then $I$ is nonzero, $d / d Y(I) \subseteq I$, but $R \cap I=0$. Furthermore $I[X] \subseteq R[[Y]][X]=\bar{A}_{1}(R)$ is a nonzero ideal of $\bar{A}_{1}(R)$ but $I[X] \cap R=0$.

PROOF. It is clear that $R$ is a subring of $k\left[x, t \mid t^{2}=0\right]$ which does not contain $x$. Note that $d / d Y\left(t e^{x Y}\right)=t x e^{x Y}$, so that $t e^{x Y}, t x e^{x Y}, t x^{2} e^{x Y}, \ldots$ generate $I$ as an ideal of $R[[Y]]$. Now suppose $r \in R \cap I$. Then $r=\sum_{i=0}^{n} t x^{i} e^{x Y} f_{i}$, where $f_{i} \in R[[Y]]$. Consider this as an equation in $k\left[x, t \mid t^{2}=0\right][[Y]]$. Then $r e^{-x Y}=\sum_{i=0}^{n} x^{i}\left(t f_{i}\right)$. Since $t^{2}=0, t f_{i}$ does not involve $x$, so that the right-hand side of this equation has bounded $x$-degree. If $r$ is nonzero, the left-hand side has unbounded degree in $x$. Thus $r=0$ and $I \cap R=0$.

The last statement is clear.

$\bar{A}_{n}(R)$ is defined inductively to be

$$
\bar{A}_{1}\left(\bar{A}_{n-1}(R)\right)=R\left[\left[Y_{1}\right]\right]\left[X_{1} ; d / d Y_{1}\right] \cdots\left[\left[Y_{n}\right]\right]\left[X_{n} ; d / d Y_{n}\right] .
$$

Now let $L$ be a Lie algebra over the field $k$ and let $R$ be a $k$-algebra. Then $L$ is said to act as derivations on $R$ if there is a Lie algebra map $\alpha: L \rightarrow \operatorname{Der}_{k} R$, 
where $\operatorname{Der}_{k} R$ is the Lie algebra of $k$-linear derivations on $R$. The smash product, $R \# U(L)$, of $R$ with $U(L)$ the universal enveloping algebra of $L$, is the $k$-space $R \otimes_{k} U(L)$. It becomes a $k$-algebra under the multiplication coming from the subalgebras $R=R \otimes 1$ and $U(L)=1 \otimes U(L)$ together with the additional rule that if $x \in L$ and $r \in R$, then $[x, r]=x r-r x=\delta_{x}(r)$, where $\delta_{x}=\alpha(x)$.

THEOREM 8. Let $R$ be an algebra over a field $k$ of characteristic zero and let $L$ be a finite dimensional solvable Lie algebra over $k$ which acts on $R$ as derivations. Then the smash product $R \# U(L)$ can be embedded in $\bar{A}_{n}(R)$, where $n=\operatorname{dim}_{k} L$.

Proof. Since $L$ is solvable, we can choose a basis $x_{1}, \ldots, x_{n}$ for $L$, where $\left\langle x_{1}, \ldots, x_{i}\right\rangle \triangleleft\left\langle x_{1}, \ldots, x_{i+1}\right\rangle$. Then $R \# U(L)=R\left[x_{1} ; \delta_{1}\right]\left[x_{2} ; \delta_{2}\right] \cdots\left[x_{n} ; \delta_{n}\right]$ is an iterated differential operator ring, where $\delta_{i+1}$ is a derivation on the ring $R\left[x_{1} ; \delta_{1}\right] \cdots\left[x_{i} ; \delta_{i}\right]$.

Now proceed by induction on $n$, the case $n=1$ being done in Theorem 4 . Letting $R_{i}=R\left[x_{i} ; \delta_{1}\right] \cdots\left[x_{i} ; \delta_{i}\right]$, note that by the inductive hypothesis, $R_{n-1}$ embeds in $\bar{A}_{n-1}(R)$. If $R_{n-1} \subseteq \bar{A}_{n-1}(R)$, then

$$
R_{n}=R_{n-1}\left[x_{n} ; \delta_{n}\right] \subseteq \bar{A}_{1}\left(R_{n-1}\right) \subseteq \bar{A}_{1}\left(\bar{A}_{n-1}(R)\right)=\bar{A}_{n}(R)
$$

In the case where $L$ is nilpotent and acts on $R$ as locally nilpotent derivations, the successive derivations $\delta_{i}$, which occur in the proof of the last theorem, are locally nilpotent. Thus we have the following result. The existence of such an embedding follows from [1, Corollary 4.4].

THEOREM 9. Let $R$ be an algebra over a field $k$ of characteristic zero and let $L$ be a finite dimensional nilpotent Lie algebra over $k$, which acts on $R$ as locally nilpotent derivations. Then the smash product $R \# U(L)$ can be embedded in $A_{n}(R)$, where $n=\operatorname{dim}_{k} L$.

EXAMPLE 10. Let $L=k x_{1}+k x_{2}$ be the solvable Lie algebra of dimension 2, with $\left[x_{2}, x_{1}\right]=x_{1}$. Assume $L$ acts on the $k$-algebra $R$, where the action of $x_{1}$ is given by $\delta_{1}$ and the action of $x_{2}$ is $\delta_{2}$. Then the embedding of $R^{\#} U(L)$ in $\bar{A}_{2}(R)$ is given by $r \in R$ goes to $\sum_{i=0}^{\infty} \sum_{j=0}^{\infty}\left(\delta_{1}^{i} \delta_{2}^{j}(r) / i ! j !\right) Y_{1}^{i} Y_{2}^{j}, x_{2}$ goes to $X_{2}$, and $x_{1}$ goes to $X_{1} e^{Y_{2}}$.

PROOF. Let $\bar{A}_{1}(R)$ have indeterminates $X_{1}$ and $Y_{1}$, while $\bar{A}_{1}\left(R\left[x_{1} ; \delta_{1}\right]\right)$ and $\bar{A}_{1}\left(\bar{A}_{1}(R)\right)$ have indeterminates $X_{2}$ and $Y_{2}$. Also let $\varepsilon_{1}: R\left[x_{1} ; \delta_{1}\right]\left[x_{2} ; \delta_{2}\right] \rightarrow$ $\bar{A}_{1}\left(R\left[x_{1} ; \delta_{1}\right]\right)$ and $\varepsilon_{2}: R\left[x_{1}, \delta_{1}\right] \rightarrow \bar{A}_{1}(R)$ be the maps given in the proof of Theorem 8 . We need to consider the composite

$$
R\left[x_{1} ; \delta_{1}\right]\left[x_{2} ; \delta_{2}\right] \stackrel{\varepsilon_{2}}{\longrightarrow} \bar{A}_{1}\left(R\left[x_{1} ; \delta_{1}\right]\right) \stackrel{\bar{A}_{1}\left(\varepsilon_{1}\right)}{\longrightarrow} \bar{A}_{1}\left(\bar{A}_{1}(R)\right),
$$

where $\bar{A}_{1}\left(\varepsilon_{1}\right)$ acts like $\varepsilon_{1}$ on $R\left[x_{1} ; \delta_{1}\right]$ and sends $X_{2}$ and $Y_{2}$ to themselves. Thus if $r \in R$,

$$
r \rightarrow \sum_{j=0}^{\infty}\left(\delta_{2}^{j}(r) / j !\right) Y_{2}^{j} \rightarrow \sum_{i=0}^{\infty} \sum_{j=0}^{\infty}\left(\delta_{1}^{i} \delta_{2}^{j}(r) / i ! j !\right) Y_{1}^{i} Y_{2}^{j}
$$

Also

$$
x_{1} \rightarrow \sum_{j=0}^{\infty}\left(\delta_{2}^{j}\left(x_{1}\right) / j !\right) Y_{2}^{j}=\sum_{j=0}^{\infty}\left(x_{1} / j !\right) Y_{2}^{j}=x_{1} e^{Y_{2}} \rightarrow X_{1} e^{Y_{2}}
$$

and $x_{2} \rightarrow X_{2} \rightarrow X_{2}$. 
We write $\operatorname{dim} R$ for the right Goldie dimension of the $\operatorname{ring} R$. It was shown by $R$. Shock [5] that $\operatorname{dim} R[x]=\operatorname{dim} R$. We extend this in Theorem 15 to show that if $R$ is a $\mathbf{Q}$-algebra and $\delta$ a locally nilpotent derivation on $R$, then $\operatorname{dim} R[x, \delta]=\operatorname{dim} R$. This was also shown to be true when $R$ is a semiprime Goldie ring by G. Sigurdsson [6].

If $I$ is a right ideal of $R$, we write $I[x ; \delta]$ for $I R[x ; \delta]$ which is a right ideal of $R[x ; \delta]$. We use the symbol $\overline{\mathbf{r}}$ to denote right annihilators. Thus $\overline{\mathbf{r}}_{R}(t)$ is the right annihilator of $t$ in $R$. It is convenient to isolate the argument in the following lemma.

LEMMA 11. Let $S=R[x ; \delta]$ be a differential operator ring and let $I \subseteq R[x ; \delta]$ be a nonzero right ideal.

(i) If $h=c_{0}+c_{1} x+\cdots+c_{n} x^{n}$, with $c_{n} \neq 0$, is a nonzero element of I of minimal degree $n$, then $\overline{\mathbf{r}}_{S}(h)=\overline{\mathbf{r}}_{R}\left(c_{n}\right)[x ; \delta]$.

(ii) If $f=a_{0}+\cdots+a_{n} x^{n}$ and $g=b_{0}+\cdots+b_{m} x^{m}$ are nonzero elements of $I$ with $a_{n}, b_{m} \neq 0$ and $n+m$ minimal subject to $f R[x ; \delta] \cap g R[x ; \delta]=0$, then $\overline{\mathbf{r}}_{S}(f)=\overline{\mathbf{r}}_{R}\left(a_{n}\right)[x ; \delta]$.

PROOF. (i) Note that if $r \in R$, then $x^{t} r=r x^{t}+l$, where $l$ involves only lower points of $x$. Thus $h r=c_{n} r x^{n}+\left(\right.$ lower degree terms). Now if $r \in \overline{\mathbf{r}}_{R}\left(c_{n}\right)$, then $h r \in I$ and $h r$ has degree less than that of $h$. Hence $h r=0$, and $\overline{\mathbf{r}}_{R}\left(c_{n}\right)[x ; \delta] \subseteq \overline{\mathbf{r}}_{S}(h)$.

Conversely, let $k=b_{0}+b_{1} x+\cdots+b_{t} x^{t} \in \overline{\mathbf{r}}_{S}(h)$. Note that $h k=c_{n} b_{t} x^{n+t}+($ lower degree terms). Since $h k=0$, we find that $b_{t} \in \overline{\mathbf{r}}_{R}\left(c_{n}\right)$. Hence $h b_{t} x^{t}=0$, giving that $b_{0}+\cdots+b_{t-1} x^{t-1} \in \overline{\mathbf{r}}_{S}(h)$. Repeating this argument gives that $b_{i} \in \overline{\mathbf{r}}_{R}\left(c_{n}\right)$ for each $i$. Thus $k \in \overline{\mathbf{r}}_{R}\left(c_{n}\right)[x ; \delta]$ and $\overline{\mathbf{r}}_{S}(h) \subseteq \overline{\mathbf{r}}_{R}\left(c_{n}\right)[x ; \delta]$.

(ii) $f R[x ; \delta]$ is a right ideal of $R[x ; \delta]$ and since $n+m$ is minimal subject to $f R[x ; \delta] \cap g R[x ; \delta]=0$, it follows that $f$ is an element of $f R[x ; \delta]$ of minimal degree. Now part (i) applies to give the result.

The following lemma forms part of the proof of Shock's theorem and is included for the sake of completeness.

LEMMA 12. Let $U \subseteq R$ be a uniform right ideal. Then $U[x] \subseteq R[x]$ is again a uniform right ideal.

PROOF. If $u[x]$ is not a uniform right ideal, choose $f, g \in U[x] \backslash 0$, such that $\operatorname{deg} f+\operatorname{deg} g$ is minimal subject to $f R[x] \cap g R[x]=0$. Let $f=a_{0}+\cdots+a_{n} x^{n}$, with $a_{n} \neq 0$, and let $g=b_{0}+\cdots+b_{m} x^{m}$, with $b_{m} \neq 0$. We can assume $m \leq n$ and, since $U$ if uniform, we may further assume $a_{n}=b_{m}$. Then by Lemma 11(ii), $\overline{\mathbf{r}}_{R[x]}(f)=\overline{\mathbf{r}}_{R}(a)[x]=\overline{\mathbf{r}}_{R[x]}(g)$.

Now consider $h=f-g x^{n-m} \in U[x]$. If $h=0$ then $f=g x^{n-m} \in f R[x] \cap g R[x]$ gives a contradiction. Thus $h \neq 0$. Note that $\operatorname{deg} h<\operatorname{deg} f$, so by the minimality of sum of the degrees, $h R[x] \cap g R[x] \neq 0$. Now choose $\alpha, \beta \in R[x]$ so that $h \alpha=g \beta \neq 0$. Thus $\left(f-g x^{n-m}\right) \alpha=g \beta$ or $f \alpha=g\left(x^{n-m} \alpha+\beta\right)$. If $f \alpha=0$ then $g \alpha=0$ so that $h \alpha=0$. Since $h \alpha \neq 0$ we conclude that $0 \neq f \alpha=g\left(x^{n-m} \alpha+\beta\right)$, which is a contradiction.

The next result is well known.

LEMMA $13 . \operatorname{dim} R[x ; \delta] \geq \operatorname{dim} R$ for any differential operator ring $R[x ; \delta]$.

PROOF. If $I_{1}, \ldots, I_{n}$ is an independent set of right ideals of $R$, then $I_{1}[x ; \delta], \ldots$, $I_{n}[x ; \delta]$ forms an independent set of right ideals of $R[x ; \delta]$. 
THEOREM 14. $\operatorname{dim} A_{1}(R)=\operatorname{dim} R$ for any ring $R$.

ProOF. From Lemma 13, we may assume $\operatorname{dim} R$ is finite. Let $\operatorname{dim} R=n$ and let $E=U_{1}+\cdots+U_{n}$ be a direct sum of $n$ uniform right ideals of $R$, which is essential as a right ideal. We claim that $E A_{1}(R)=U_{1} A_{1}(R)+\cdots+U_{n} A_{1}(R)$ is a direct sum of $n$ uniform right ideals of $A_{1}(R)$ which is essential as a right ideal. Indeed $E A_{1}(R)$ is essential as an $R$-submodule of $A_{1}(R)$ and hence also as a right ideal. It remains to show that if $U \subseteq R$ is a uniform right ideal of $R$, then $U A_{1}(R)$ is a uniform right ideal of $A_{1}(R)$. This is achieved by a variation on the argument in Lemma 12.

Suppose $U A_{1}(R)$ is not uniform. Then let $f=\sum_{i=0}^{n} f_{i} X^{i}, g=\sum_{j=0}^{k} g_{j} X^{j}$, with $f_{i}, g_{j} \in U[Y]$, be nonzero elements of $U A_{1}(R)$ such that $f A_{1}(R) \cap g A_{1}(R)=0$. Fix $n$ and $k$ so that $n+k$ is minimal and assume $n \geq k$. Note that if $h(Y) \in R[Y]$ then $X^{n} h(Y)=h(Y) X^{n}+($ lower powers of $X)$. Also $U[Y]$ is a uniform ideal of $R[Y]$ by Lemma 12. Thus we may further assume $f_{n}=g_{k}$. Lastly we may also assume that the $Y$-degree of $f_{n}$ is minimal in $f_{n} R[Y]$. Now let $a \in R$ be the leading coefficient of $f_{n} \in R[Y]$. Then $\overline{\mathbf{r}}_{A_{1}(R)}(f)=\overline{\mathbf{r}}_{R[Y]}\left(f_{n}\right) A_{1}(R)$ by Lemma 11(ii). But $\overline{\mathbf{r}}_{R[Y]}\left(f_{n}\right)=\overline{\mathbf{r}}_{R}(a) R[Y]$ by Lemma $11(\mathrm{i})$, so that $\overline{\mathbf{r}}_{A_{1}(R)}(f)=\overline{\mathbf{r}}_{R}(a) A_{1}(R)$. Similarly, $\overline{\mathbf{r}}_{A_{1}(R)}(g)=\overline{\mathbf{r}}_{R}(a) A_{1}(R)$. Since $R$ commutes with $X$ it follows that $\overline{\mathbf{r}}_{R}(a) A_{1}(R)$ annihilates $g X^{n-k}$.

Let $h=f-g X^{n-k}$. We may assume $h \neq 0$ since otherwise $f=g X^{n-k}$. Now since $X$-deg $h<X$-deg $f$ and $n+k$ is minimal, we see that $h A_{1}(R) \cap g A_{1}(R) \neq 0$. Choose $\alpha, \beta \in A_{1}(R)$ so that $0 \neq h \alpha=g \beta$. This implies that $f \alpha=g\left(X^{n-k} \alpha+\beta\right)$. If $f \alpha=0$ then $h \alpha=0$, since $\overline{\mathbf{r}}_{A_{1}(R)}\left(g X^{n-k}\right) \supseteq \overline{\mathbf{r}}_{A_{1}(R)}(g)=\overline{\mathbf{r}}_{A_{1}(R)}(f)$. Thus $0 \neq f \alpha=g\left(X^{n-k} \alpha+\beta\right)$ which contradicts the assumption that

$$
f A_{1}(R) \cap g A_{1}(R)=0 .
$$

Finally, we combine our methods to prove

THEOREM 15. Let $R$ be a Q-algebra and let $\delta$ be a locally nilpotent derivation on $R$. Then $\operatorname{dim} R[x ; \delta]=\operatorname{dim} R$.

PROOF. We use the notation of Theorem 2 and combine that result with Lemma 13 to conclude that $\operatorname{dim} \tilde{R} \leq \operatorname{dim} \tilde{R}[X] \leq \operatorname{dim} \tilde{R}[X][Y]$. But $\tilde{R} \simeq R, \tilde{R}[X] \simeq R[x ; \delta]$, and $\tilde{R}[X][Y]=A_{1}(R)$. Thus by Theorem 14, equality holds above.

ACKNOWLedgements. The author would like to thank D. S. Passman for valuable suggestions, and in particular for Example 7. The author also thanks W. Chin for helpful conversations.

NOTE ADDED IN PROOF. Two papers are to appear on related subjects, Uniform rank over differential operator rings and Poincaré-Birkhoff-Witt extensions, Pacific J. Math., by A. Bell and and K. Goodearl, and Goldie dimension of differential operator rings, Comm. Algebra, by P. Grzeszczuk. Both these papers include additional situations in which $\operatorname{dim} R=\operatorname{dim} R[x ; \delta]$. The first contains another proof of Theorem 14, with the result stated for any induced module, and gives an example which shows that Theorem 15 fails if $\delta$ is not assumed locally nilpotent. 


\section{REFERENCES}

1. R. J. Blattner and S. Montgomery $A$ duality theorem for Hopf module algebras, J. Algebra 95 (1985), 153-172.

2. W. Borho, P. Gabriel, and R. Rentschler, Primideale in Einhullenden auflosbarer Lie-Algebren, Lecture Notes in Math., vol. 357, Springer-Verlag, Berlin and New York, 1973.

3. T. Masuda, Duality for a differential crossed product and its periodic cohomology, C. R. Acad. Sci. Paris Sér. I 301 (1985), 551-553.

4. Y. Nouaze and P. Gabriel, Idéaux premiers de l'algèbre enveloppante d'une algèbre de Lie nilpotente, J. Algebra 6 (1967), 77-99.

5. R. C. Shock, Polynomial rings over finite dimensional rings, Pacific J. Math. 42 (1972), 251257.

6. G. Sigurdsson, Differential operator rings whose prime factors have bounded Goldie dimension, Arch. Math. (Basel) 42 (1984), 348-353.

Department of Mathematics, University of Wisconsin, Madison, Wisconsin 53706

Current address: Department of Mathematics, University of Utah, Salt Lake City, Utah 84112 